\title{
The breakthrough of a quantum chemist by classical dynamics: Martin Karplus and the birth of computer simulations of chemical reactions
}

\author{
Daniele Macuglia ${ }^{1,2}$, Benoît $\operatorname{Roux}^{3, a}{ }_{\mathbb{D}}$, and Giovanni Ciccotti ${ }^{4,5,6}$ \\ 1 Department of History of Science, Technology and Medicine, Peking University, Beijing 100871, China \\ 2 Neubauer Collegium for Culture and Society, University of Chicago, Chicago, IL 60637, USA \\ 3 Department of Biochemistry and Molecular Biology, University of Chicago, Chicago, IL 60637, USA \\ 4 Department of Physics, University of Rome, "La Sapienza", 00185 Rome, Italy \\ 5 IAC-CNR, Institute for the Application of Computing "M. Picone", National Research Council, 00185 Rome, Italy \\ ${ }^{6}$ School of Physics, University College Dublin, Belfield, Dublin 4, Ireland
}

Received 19 December 2020 / Accepted 26 March 2021 / Published online 10 June 2021

(C) The Author(s) 2021

\begin{abstract}
Martin Karplus' research - a time when, rather unexpectedly, he approached the problem of reactive collisions using a quasiclassical approximation with the aid of computer technologies. This marked a substantial departure from the quantum-chemical studies of nuclear magnetic resonance that had, until then, dominated his work. The historical perspective outlined by George Schatz, as well Karplus' own biography, partly frames the contours of this remarkable period in the history of theoretical chemistry. Yet, the available historical literature is not sufficiently complete to allow us to understand Karplus' transition from nuclear magnetic resonance to reaction dynamics. In this article, we discuss the intellectual ground on which Karplus operated around 1964, further commenting on the relevance of his quantum and quasiclassical studies and pondering how Karplus' approach eventually led to his interest in the simulation of complex biomolecules.
\end{abstract}

\section{Introduction}

From his earliest school years, biology represented an irresistible interest for Martin Karplus (born 1930), his "first love ${ }^{1}$." Yet, notwithstanding this interest in biology, he followed a different trajectory in the early stages of his academic training. "I had concluded that to approach biology at a fundamental level (to understand life), a solid background in chemistry, physics, and mathematics was imperative, and so I enrolled in the Program in Chemistry and Physics ${ }^{2}$." The year was 1947, and the school was Harvard college, where Karplus performed his undergraduate studies. At this stage, we can already detect two important aspects of his life: his interest in the biological sciences and his tendency toward taking unexpected paths.

\footnotetext{
1 Karplus (2006, p. 1). We also relied on Karplus (2020), which had just appeared prior to finalizing our article. The two publications, however, essentially do not differ regarding the kinds of questions we sought to tackle.

2 Karplus (2006, p. 12).

a e-mail: roux@uchicago.edu (corresponding author)
}

In 1950, Karplus moved to the California Institute of Technology (Caltech) for his graduate studies. He began to work as a graduate student in biology under Max Delbrück (1906-81), but he switched to chemistry after their relationship became strained ${ }^{3}$. He then started to work under John Kirkwood (1907-59), embarking on research into the charge fluctuations of proteins, but finally joined Linus Pauling's (1901-94) laboratory to focus on an $a b$ initio approach to the bifluoride ion after Kirkwood left for Yale University in $1951^{4}$. Connections to biology, either direct or indirect, remained in the air but were as yet undeveloped. Worth noting, from a biological perspective, was the presence of two remarkable scientists in Pauling's group, scholars who would go on to make substantial contributions to the biological sciences: chemist Leslie Orgel (1927-2007), who was active in biology as a theorist of the origin of life, as well as biologist and biophysicist Alexander Rich (1924-2015), who made numerous seminal contri-

\footnotetext{
3 Karplus (2006, pp. 15-16).

4 Harvard sources report that Karplus is considered Pauling's most brilliant student. See Ireland, Corydon, 2017. I had the conviction that my ideas were correct. The Harvard Gazette. April 21. Source: https://news.harvard.edu/gazette/story/2017/04/harvards -martin-karplus-looks-back-on-path-to-nobel-prize/.
} 
butions on the structure and function of nucleic acids ${ }^{5}$. "Interacting with them," remarked Karplus while referring to all members of Pauling's group, "was a wonderful part of my Caltech education ${ }^{6}$." Karplus found himself operating in a multifaceted environment that offered myriad approaches to chemistry, physics, and biology.

Soon after completing his Ph.D. in 1953, Karplus embarked on a two-year postdoctoral stay at the University of Oxford, where he worked as a National Science Foundation (NSF) Postdoctoral Fellow under chemist and applied mathematician Charles Coulson (1910-74). There, he collaborated with Simon Altmann (born 1924), who helped to improve his knowledge of group theory. Karplus also made contact with William Lipscomb (1919-2011), then famous for his work on boron chemistry and nuclear magnetic resonance (NMR), and who was then becoming active in research on X-ray crystallography and the structure and behavior of enzymes ${ }^{7}$. Karplus did not actually work on any concrete chemical problems at Oxford, but he did try to concoct ideas that could be useful not just for chemists but for an interdisciplinary community of scholars. His connections with chemist Donald Hornig (1920-2013) and physicist Maurice Pryce (1913-2003) became a triggering force behind his decision to embark on NMR studies. Notably, here we see another unexpected change in direction.

In 1955, Karplus started his career as a faculty member of the University of Illinois at Urbana-Champaign, where he focused a good deal on his research "on theoretical methods for relating nuclear and electron spin magnetic resonance parameters to the electronic structure of molecules ${ }^{8}$." Urbana-Champaign, especially in the mid-1950s, was a leading institution for the application of NMR to chemistry. During those years, Herbert Gutowsky (1919-2000) and Charles Slichter (19242018) became founding fathers in these new approaches to chemistry. As explained by Karplus, the Department of Chemistry was going through some major renovations, with a number of vacancies to fill. It was Pauling who wrote a recommendation letter for Karplus in support of his candidacy. The young scholar filled one of those vacancies and upon his hiring received some funds to conduct his research. Soon enough Karplus found himself operating within a stimulating environment, full of opportunities. As he remarked, "the presence of four new instructors-Rolf Herber, Aron Kupperman, Robert Ruben, and me- plus other young scientists on the faculty, such as Doug Applequist, Lynn Belford, and E.J. Corey, led to a very interactive and congenial atmosphere ${ }^{9}$."

\footnotetext{
5 Orgel (1973), Rich (2019).

6 Karplus (2006, p. 16).
}

7 Lipscomb was awarded the 1976 Nobel Prize in Chemistry "for his studies on the structure of boranes illuminating problems of chemical bonding." See https://nobelprize. org/prizes/chemistry/1976/summary/.

8 Karplus (2006, p. 19).

9 Karplus (2006, p. 19).
The 1950s represented a very special time in theoretical chemistry, witnessing the blossoming of quantum approaches to chemistry, and especially those that targeted the electronic structure of molecules. In 1926, Erwin Schrödinger (1887-1961) applied to the hydrogen atom the time-independent form of the equation that bears his name, he solved it and contributed to the new and coherent approach of atomic physics that substituted, with quantum mechanics, the old quantum theory ${ }^{10}$. Shortly later, in 1927, Walter Heitler (1904-81) and Fritz London (1900-54) used a variational approach to introduce the first approximate quantum treatment of chemical bonds, also known as the valence bond method $^{11}$. This approach was further developed by the physicist John Slater (1900-76) and Pauling. The latter introduced the concepts of resonance and orbital hybridization, expanding from early concepts elaborated by Gilbert Lewis $(1875-1946)^{12}$. In recognition of these results, in 1954 Pauling was awarded the Nobel Prize in Chemistry "for his research into the nature of the chemical bond and its application to the elucidation of the structure of complex substances ${ }^{13}$." This period thus represented a golden era in the application of quantum mechanics to chemistry.

Karplus' studies at Urbana-Champaign resulted in a particularly relevant paper that he published in the Journal of Chemical Physics in $1959^{14}$. This study was made possible thanks to the ILLIAC I digital computer, which was built at Urbana-Champaign and had become operative in 1952. Here, we note another important ingredient for understanding Karplus' scientific life: an early and unconditioned confidence in the use of computer technologies. It is clear that, from the very beginning, Karplus was open to the use of computers, thoroughly understanding their essential role in shaping new trends in scientific inquiry. In particular, the device available at Urbana-Champaign worked by processing a series of punched holes in paper tapes; it had a memory of 1000 words. "If you made a mistake," remarked Karplus, "you filled in the incorrect holes with nail polish so that you could continue the program, the output appearing on spools of paper ${ }^{15}$." Nonetheless, "Probably the most valuable aspect of having a program for this type of simple calculation [...]," he stressed, "was that once the program was known to be correct, a large number of calculations could be performed without having to worry about arithmetic mistakes ${ }^{16}$." This marked an unprecedented change in the history of science: the use of computers allowed for a remarkable shift from the formulation of scientific laws, with lim-

\footnotetext{
10 Schrödinger (1926).

11 Heitler and London (1927).

12 Lewis (1916).

13 See www.nobelprize.org/prizes/chemistry/1954/summary/. It is worth noting that Karplus had only just graduated with his $\mathrm{PhD}$ under Pauling's mentorship the previous year.

14 Karplus (1959a).

15 Karplus (2006, p. 20).

16 Karplus (2006, p. 20).
} 
ited computational capabilities, to the formulation and effective solution of the equations that those scientific laws demanded ${ }^{17}$.

It was just before publishing the aforementioned 1959 paper that Karplus attended a lecture by organic chemist Raymond Lemieux (1920-2000). The lecture was on the conformations of acetylated sugars, and Karplus found it particularly stimulating. Lemieux reported having measured NMR spin-spin vicinal coupling J-constants between a pair of atoms separated by three chemical bonds, and he noted that there appeared to be a dihedral angle dependence, exactly as Karplus imagined and later reported in his article ${ }^{18}$. The simple mathematical expression of the three-bond J-coupling constants, in terms of the dihedral angles, eventually became known as the "Karplus equation" ${ }^{19}$." Elias James Corey (born 1928), in that same year, developed what is believed to be the first application to organic chemistry of Karplus' results ${ }^{20}$.

Karplus' work at Urbana-Champaign took place within a vibrant and prolific framework. "I continued to work on problems in NMR and ESR (electron spin resonance) because new areas of chemistry were being studied by these spectroscopic methods and it seemed worthwhile to try to provide insights from theoretical analyses of some of these applications ${ }^{21}$." At UrbanaChampaign, Karplus engaged in a dialogue connected to the proliferation of these applications, a conversation then developing with the participation of other remarkable chemists. Yet, the "chemistry department at Illinois was rigidly separated into divisions, which had a semiautonomous existence ${ }^{22}$;" it was around computer technologies, evidently, that scholars started to work in a more interdisciplinary way. This observation emphasizes the remarkable impacts that computers were having (and are still having), not just in terms of improved computational capabilities, but also in favoring new connections among scientific fields and subfields, allowing scientists to transcend boundaries that had traditionally categorized various stages in the history of science and technology ${ }^{23}$.

At the beginning of the 1960s, it looked like Karplus was off to a productive career in the application of NMR to theoretical chemistry. As it happened, however, he made another unexpected maneuver. In 1964 and 1965, he co-published research articles that took him in a completely different direction, toward a quasiclassical

17 Macuglia et al. (2020, pp. 73-74).

18 Karplus (2006, p. 20).

19 Karplus (2006, p. 20). See also Conroy (1960). The notion of Karplus equation was not free from criticism, as some scientists were concurrently incurring some deviations of the measurements from what had been predicted by the theory. Karplus replied to such criticism with a 1963 paper. See Karplus (1963).

20 Bradshaw et al. (1959).

21 Karplus (2006, p. 21). See also Karplus (1959b, 1960a, b).

22 Karplus (2006, p. 20).

23 Macuglia et al. (2020, pp. 64, 74). approach to collision-reaction dynamics ${ }^{24}$. By this time, Karplus had already moved to Columbia University (in the fall of 1960), where he made consistent use of the local computer facilities and considerably changed his research topic. Interestingly, as we shall see, the most remarkable shifts in Karplus' way of thinking all coincide with changes in his academic setting: Caltech and an $a b$ initio approach to the bifluoride ion, Oxford and the time of creative thinking, Urbana-Champaign and NMR, Columbia and reaction dynamics. Not only did Karplus make flexible changes to his research topics, but he was able to skillfully contribute to the new research lines on which he focused. Upon realizing that he had made significant contributions to a given area of study, he would suddenly embark on a trek into a new, virgin field. And his changing circumstances inevitably relied on computer technologies. Eventually, because of computers and the vast experience he had accumulated in chemistry and physical chemistry, Karplus was able to effectively turn to the biological sciences, exactly as he had envisioned when still a young man at Harvard.

At Columbia, Karplus first joined the IBM Watson Scientific Laboratory, where he devoted himself to the study of the $\left(\mathrm{H}, \mathrm{H}_{2}\right)$ reaction in the Born-Oppenheimer approximation. Yet before taking this new position in the Laboratory, he requested a tenured position at the Chemistry Department, which he obtained, thus assuring himself a position at Columbia. This turned out to be remarkably intuitive, as IBM closed down that particular branch of their Watson Scientific Laboratory in 1970. While Karplus was not in New York anymoreand therefore unaffected by the closing - this situation further cemented Karplus' sense that a university provided a much safer environment than a private research laboratory, which could refocus its interest or even shut down unexpectedly, depending on the corporate funding situation. As he stressed in his own autobiography, Karplus has always believed that fundamental research run by big industries may not always provide a stable trajectory to follow, especially for young scientists in the early stages of their careers ${ }^{25}$. For this reason, he has always advised young researchers to take care when leaving academia to venture into privately run laboratories.

Having said that, the IBM Watson Scientific Laboratory exemplified a truly vibrant research center, especially due to the presence of physicists such as Llewellyn Thomas (1903-92), Erwin Hahn (1921-2016) and Seymour Koenig (1927-2018). The Laboratory was directed by Wallace Eckert (1902-71), an astronomer focusing on the three-body problem by means of computing technologies ${ }^{26}$. Karplus himself was focusing on a three-body problem, considering the intermediate $\mathrm{H}_{3}$ state of the $\left(\mathrm{H}, \mathrm{H}_{2}\right)$ reaction, so he found in the laboratory prolific ground to grow. At Columbia, in addi-

\footnotetext{
24 The two mentioned articles will be analyzed in the next section: "1964: the unexpected rise of quasiclassical and classical approaches."

25 Karplus (2006, p. 22).

26 Karplus (2006, p. 22).
} 
tion, he was involved in teaching and research, and he could rely on the support of a group of postdocs. The Chemistry Department turned out to be a good fit, an environment where Karplus could work sideby-side with scholars such as physical chemist George Fraenkel (1921-2009), organic chemist Ronald Breslow (1931-2017), and Richard Bersohn (1925-2003), who was active in chemical kinetics. The laboratory came equipped with an IBM 650, "an early digital computer, which was much more useful than the ILLIAC because of its greater speed, larger memory, and simpler (card) input ${ }^{27}$." It was primarily thanks to this computer that Karplus was able to study the $\left(\mathrm{H}, \mathrm{H}_{2}\right)$ reaction at a fundamental level, as we will explain in the next section.

Some points from this timeline seem essential to the framing of Karplus' own background. First, there was his unconditional attraction to biology, his first love, an ingredient which did not become evident at Columbia, yet had always been there, in the background, since his youth. This love made a resurgence during the next stage, the professorship at Harvard (which he started in 1966), where Karplus shifted his research focus, venturing into biomolecules.

Another important ingredient to Karplus' success was his flexibility and versatility. His ability to adapt to changing situations, in order to rework his professional connections, took him in different research directions. If he felt that a given topic was not mature enough or that new substantial contributions couldn't be made, he would change topics and venture onto a new topic that held the promise of groundbreaking developments. As a matter of fact, when he realized that he had reached his goals in NMR studies, he eventually decided to focus on chemical reactions. This ability to find promising new directions, to readapt to different contexts, and to begin new research topics is not a common feature among contemporary scientists. For this reason, Karplus' approach is unusual from the perspective of scientific knowledge production. Most research scientists tend to follow a given trajectory and keep working out all the fine details of a given research topic. With Karplus, we notice early interests in NMR coupling constants, reaction kinetics, and eventually biomolecules.

The third remarkable aspect of Karplus' approach was his conscious and decisive use of computing technologies when attacking chemical problems. This, indeed, represents the common denominator of all his research projects. This feature first manifested itself at UrbanaChampaign and became even more decisive when Karplus moved to Columbia, where he clearly understood that, with the use of computers, he could study chemical reactions in a quantitatively groundbreaking way. By exploiting the power of computers, in fact, he was able to obtain a reasonable and accurate description of the energy surface for the $\left(\mathrm{H}, \mathrm{H}_{2}\right)$ reaction in the Born-Oppenheimer approximation, and then make a substantial contribution to the description of the major kinetic and dynamic properties of this reaction. This

$\overline{27}$ Karplus (2006, p. 22). eventually constituted one of his most remarkable contributions to both theoretical and experimental chemistry.

\section{1964: the unexpected rise of quasiclassical and classical approaches}

Karplus has been the kind of scientist who, from his youth, was interested in developing those off-track and unexplored areas of research that might possibly produce major, unexpected breakthroughs. Through creative thinking, he was keen to find new ways of using tools and techniques that already existed, producing results that could play a role in inaugurating new directions of research. This was something that already happened at Urbana-Champaign with his NMR studies, as we have seen in the case of the Karplus equation, a contribution to spectroscopic analysis by theoretical chemistry. Yet, after having achieved this result, Karplus felt that he had obtained most of the essential results - and he did not intend to pursue this subject any further. As he noted in an interview with us, "I felt that I should change my focus of research every five years or so, because I understood a given area and my excitement in something possibly new in those areas was no longer there." This testimonial reinforces what we highlighted in the previous section, in our discussion of Karplus' need to keep changing focus and finding new research projects. We believe the present funding situation hinders this "diversity of focus," a mindset increasingly difficult to maintain amid the rigid and specialized career paths that scholars tend to follow nowadays. Today, in addition, an overcrowded academic arena encouragesor forces - many young scholars to leave the academic realm and work in the private sector, where they oftentimes face the kinds of pitfalls we identified in the previous section. The new generation of scientists therefore operates in a considerably different environment - and perhaps a more fundamentally unstable one - than that in which Karplus was active when creating his professional path.

The results Karplus got with NMR were instrumental for allowing his transition to Columbia. There, collision reaction dynamics represented a new field that promised the possibility of remarkable discoveries. Another point worth mentioning: Karplus possessed an ability to assume a certain amount of risk within his research; he remained ambitious and confident, forever seeking new research lines to follow - as happened with the trajectories computed by the reactants on which he focused when arriving at Columbia.

When he approached reaction kinetics, Karplus was already an expert quantum chemist who understood that, without computers, there would be no opportunities for substantial scientific advances. With a good computer, it was possible to compute a fairly accurate nuclear potential energy surface by which, in turn, he could focus on the scattering of the $\left(\mathrm{H}, \mathrm{H}_{2}\right)$ exchange reaction, essentially one of the simplest and most funda- 
mental chemical reactions. The need for a good potential energy surface for $\mathrm{H}$ and $\mathrm{H}_{2}$ was a critical ingredient in this story. Only an accurate potential could produce useful and meaningful results, which could then be validated by a direct comparison with experimental measurements.

To compute the nuclear potential energy surface, it is mathematically possible to isolate the electronic motion from the nuclear motion. In other words, one solves the electronic part with fixed nuclei first. Then, given that nuclei are heavy entities, one can follow, within the adiabatic approximation, the nuclear motion, assuming the electrons remain in the ground state. The ground electronic state then provides the potential energy surface for the nuclei, whose motion takes place in the average field created by the electrons. From this perspective, the Hamiltonian of the whole system (electrons plus nuclei) is reduced to the simpler Hamiltonian for the nuclei, with an effective potential corresponding to the electronic ground state. The potential obtained from this approximation is known as the Born-Oppenheimer energy surface. The potential depends parametrically only on the positions of the nuclei; the electrons do not appear explicitly. Given the fact that the nuclei are relatively heavy, and assuming that the relative distances among them remains larger than the de Broglie wavelength associated with each nucleus, it is thus possible to approximate the dynamical evolution of the nuclei using Newton's classical equations of motion.

Here, we arrive at the starting point for Karplus' own research. Collision-reaction dynamics can be approached as the reaction of nuclei treated as classical entities under the influence of the quantum Born-Oppenheimer potential (originating from the electrons in their groundstate wavefunction). Of course, some aspects of Newton's classical equations of motion are really inadequate for quantum particles. For example, a classical system can rest at the minimum of the potential energy surface with no kinetic energy. However, this classical picture of the "ground state" with minimum total energy is completely inconsistent with quantum mechanics. The true ground state of the nuclei cannot be allowed to localize at the minimum of the Born-Oppenheimer potential. A small quantum correction, the so-called zero-point energy, has to be introduced to account. Therefore, while the trajectories are based on classical mechanics, the result is better referred to as "quasiclassical."

"Given the great difference between the time required for generating an accurate semi-empirical energy function for the $\left(\mathrm{H}, \mathrm{H}_{2}\right)$ exchange reaction and generating an accurate purely quantum mechanical energy surface," Karplus remarked, "it was clear that for larger systems we had to use the former ${ }^{28}$." He then performed the quantum calculation of the potential field of the nuclei and a quasiclassical study of the trajectories of the scattering in the $\mathrm{H}+\mathrm{H}_{2} \rightarrow \mathrm{H}_{2}+\mathrm{H}$ reaction. According to this framework, one starts off with an isolated $\mathrm{H}$ atom and an isolated $\mathrm{H}_{2}$ molecule that interact, react, and form an intermediate $\mathrm{H}_{3}$ molecule. This

28 Interview with the authors. intermediate product is unstable, eventually decaying in two possible ways: $\left(\mathrm{H}, \mathrm{H}_{2}\right)$ and $\left(\mathrm{H}_{2}, \mathrm{H}\right)$. Assuming that the potential that regulates this exchangereaction is known, one can identify a series of initial conditions and follow the scattering process until the reaction takes place, then focus on the product channel that produces $\left(\mathrm{H}_{2}, \mathrm{H}\right)$. By evaluating the cross section of this reaction, one can eventually study the relative amount of $\left(\mathrm{H}_{2}, \mathrm{H}\right)$ as a reaction product.

This is essentially what Karplus was able to do in his research at Columbia. In 1964, he worked on two notable articles: a research paper which he copublished with Richard Porter (born 1932) and a short communication, co-authored with Porter and Ramesh Sharma ${ }^{29}$. The first study (1964 PK) entailed the computation of the potential energy surface for the three hydrogen atoms of the reaction $\mathrm{H}+\mathrm{H}_{2} \rightarrow \mathrm{H}_{2}+\mathrm{H}$. The second article (1964 KPS) reported the first scattering study of the $\left(\mathrm{H}, \mathrm{H}_{2}\right)$ exchange reaction based on quasiclassical trajectories on this potential energy surface. A subsequent publication by Karplus, Porter, and Sharma (1965 KPS) explained in further detail the quasiclassical procedure for the calculation of the collisionreaction dynamics of the $\left(\mathrm{H}, \mathrm{H}_{2}\right)$ reaction with activation energy ${ }^{30}$.

Acknowledging previous contributions to the field, the opening of the 1964 KPS article opening is noteworthy:

The $\left(\mathrm{H}, \mathrm{H}_{2}\right)$ exchange reaction [...] has been studied by numerous investigators. Most of these have estimated the activation energy and/or the rate constant within the framework of the activated complex formulation or closely related statistical methods. In this communication, we outline the results of a direct examination of the collision dynamics that represents the first complete quasiclassical calculation of an atom-molecule exchange reaction for a realistic potential without restrictive approximations $^{31}$.

It was evident from all the previous studies that, "Even for the simplest exchange reaction $\left(\mathrm{H}+\mathrm{H}_{2} \rightarrow\right.$ $\mathrm{H}_{2}+\mathrm{H}$ ), the best a priori theoretical treatments of the surface are as yet neither accurate nor extensive enough to permit a detailed examination of reaction dynamics ${ }^{32}$." What was important regarding the previous studies, however, was the fact that they made it clear that the $\left(\mathrm{H}, \mathrm{H}_{2}\right)$ reaction could be studied using classical mechanics, i.e., by studying the scattering process, treating the nuclei as classical objects moving in the average potential produced by the electrons. For Karplus the critical missing ingredient, preventing a

\footnotetext{
29 Porter and Karplus (1964) and Karplus et al. (1964).

30 Karplus et al. (1965).

31 As remarked by the authors of the communication, also D. R. Herschbach, A. Kuppermann, L. M. Raff, R. Gordon, and K. Tang provided assistance with the calculations Karplus (1964, p. 2033).

32 Porter and Karplus (1964, p. 1105).
} 
direct comparison with experiments, was an accurate potential energy surface.

Thus, all the necessary ingredients were already in the air; previous scientists had attempted to solve the problem of calculating a reliable potential and, with that, the quasiclassical scattering process. However, these researchers could not obtain any significant results with sufficient precision ${ }^{33}$. Karplus' merit was that of having used computers to successfully produce a reliable description of the potential for the $\left(\mathrm{H}, \mathrm{H}_{2}\right)$ exchange reaction, as well as a satisfactory description of the scattering process. At this stage we cannot yet talk of molecular dynamics (MD), though we can certainly talk of an early example of fundamental computer simulations, starting with the fundamental laws of dynamics and solving, via computer technologies, the complex mathematical equations that derive from those laws. Considering that the $\left(\mathrm{H}, \mathrm{H}_{2}\right)$ reaction is, as we have stated, a three-body problem, it would have been practically impossible to study it by means of a paper-and-pencil approach. Karplus understood that a substantial breakthrough could only be made using computers; as PK noted, in fact, "modern computing techniques reduce energy and gradient calculations for several thousand configurations to the point of triviality, particularly as compared with the complete a priori calculations ${ }^{34}$." This revelation marked the successful result of Karplus stay at Columbia, a result that impacted both his subsequent career (when he moved on to Harvard), and his research lines in theoretical chemistry. Eventually, this key point would have allowed him to return to his "first love," when he began to approach biomolecules.

It's interesting at this point to recall what had happened in the years leading up to 1964. The period from the late 1920s to the beginning of the 1940s was characterized by the first attempts to compute the potential that regulates the $\left(\mathrm{H}, \mathrm{H}_{2}\right)$ exchange reaction ${ }^{35}$. Before Karplus, the most widely used method for determining such potential was based on the equation for the energy of the polyatomic system, an approach developed in 1929 by Fritz London ${ }^{36}$. In 1931, Henry Eyring (190181) and Michael Polanyi (1891-1976) developed a new study based on valence-bond methods ${ }^{37}$. The results were not accurate, but they remained qualitatively useful in determining the development of new approaches to the problem. After a time, the renowned nuclear physicist and theoretical chemist Joseph Hirschfelder (1911-90) would join the discussion, contributing alongside Eyring, as well as Bryan Topley (1901-86) ${ }^{38}$. These

\footnotetext{
33 Schatz (2000, p. 270).

34 Porter and Karplus (1964, p. 1115).

35 Schatz (2000, p. 270).

36 Porter and Karplus (1964, p. 1106). See also London (1929), Hirschfelder et al. (1936b), Hirschfelder (1938), Ransil (1957), Snow and Eyring (1957), Kimball and Trulio (1958) and Boys and Shavitt (1959).

37 Schatz (2000, p. 270). See also Eyring and Polanyi (1931).

38 Schatz (2000, p. 270). See also Hirschfelder et al. (1936a).
}

authors attempted to develop the first classical molecular dynamics study of the reaction, a study that was actually able to determine a small fraction of the reaction trajectories, using essentially no experimental match; computing technologies were not available in the 1930s, and the authors had to basically approach the solution of the relevant equations by hand ${ }^{39}$.

The use of digital computers to study reaction trajectories first appeared in the late $1950 \mathrm{~s}^{40}$. Examples of computers available at that time include the ORDVAC, the aforementioned ILLIAC I, and the FUJIC, as well as the MUSASINO-1. The first scattering studies were still rudimentary, employing inaccurate potential energy surfaces, restricting the number of integrated trajectories, or significantly reducing the motion to one or two dimensions ${ }^{41}$. Yet, these studies made it possible to estimate the rates of a large variety of reactions ${ }^{42}$. In 1955, Shin Sato (born 1928) developed a substantial modification of the Eyring procedure, proposing a new method for drawing the potential energy surface. Sato obtained an improved potential for the systems to which it was applied, but it was difficult to justify his method on a quantummechanical ground ${ }^{43}$. PK were essentially able to refine the Eyring-Sato approach and made it consistent with a quantum-mechanical formulation, considering all overlap and multiple-exchange integrals ${ }^{44}$. In particular, the authors based their method on the nonionic valencebond approach with the inclusion of all overlap and three-center terms, which previous authors had disregarded $^{45}$. Having computed the three-body interaction, KPS were able to compute important properties of this exchange reaction, including the rate constants. This marked another salient aspect of their study, as noted by the authors:

From the cross sections, rate constants are determined by averaging over the distribution of initial conditions corresponding to particular experimental situations. Analysis of trajectories yields detailed information concerning the nature of the reactive collision (e.g., collision time, configuration in neighborhood of saddle point, dependence of reaction probability on impact parameter $)^{46}$.

Using the potential they had obtained, the authors were able to compute trajectories starting from a large sample of initial conditions. The wavefunction of the

\footnotetext{
39 Schatz (2000, p. 270).

40 Schatz lists the works by Wall et al. (1958) and Blais and Bunker (1962) as the relevant works first implying computer technologies in trajectory studies. See Schatz (2000, p. 270). 41 Schatz (2000, p. 270).

42 Porter and Karplus (1964, p. 1106). See also Weston (1959) and Shavitt (1959).

43 Porter and Karplus (1964, p. 1106), Sato (1955b) and Sato (1955c). See also Sato (1955a). Criticisms to Sato were advanced by Weston (1959).

44 Schatz (2000, p. 270).

45 Porter and Karplus (1964, p. 1115).

46 Karplus et al. (1964, p. 2033).
} 
initial $\left(\mathrm{H}, \mathrm{H}_{2}\right)$ system provided the sample of quasiclassical initial conditions. From these initial positions and velocities, they could then compute the scattering process. From there, they studied the reaction populations and their rates. This is the essence of the 1965 KPS article analyzed by Schatz (2000).

KPS influenced the further developments of the quasiclassical approach, as well as wave-packet methods for the simulation of chemical reactions and eventually of quantum mechanical theories of chemical reaction dynamics ${ }^{47}$. As Karplus remarked in a discussion with us, the quasiclassical trajectory-collision approach "was an approximation, and it gave such good results because the reaction was adiabatic, but then a full quantum mechanical treatment such as that performed by Schatz and Kuppermann was required to get exact results." It is no surprise, as George Schatz (born 1949) remarked, that the paper by KPS "is the most important early (pre-1970) piece of computational work in gas-phase chemical reaction dynamics," providing "the foundation for molecular dynamics studies of chemical reactions in condensed phases, including applications to gas-surface scattering and biomolecular simulation ${ }^{48}$."

While Porter essentially continued to pursue the same line of research, Karplus eventually embarked on something different ${ }^{49}$. Inspired by the approach he adapted for the $\left(\mathrm{H}, \mathrm{H}_{2}\right)$ reaction, he shifted his focus toward a new field that held the promise of remarkable advances. He realized that empirical energy functions were going to be necessary to scale up computational studies to large biological macromolecules, a research field he had begun to master in the 1970s. In this case, he started with an approximate potential and performed the first simulation of a protein by means of MD. Yet, as a complement to what Berni Alder (1925-2020) and Thomas Wainwright (1927-2007), as well as Aneesur Rahman (1927-87) and Frank Stillinger (born 1934) had done, Karplus avoided focusing on generic atomic and molecular motions in liquids and other dense systems; instead, he was specifically interested in the motions of the very atoms forming a protein. In other words, he delved into the fundamental atomic motions in the interior of a protein, the motions essential for the chemical activity of biological macromolecules. To put this interest in a broader context, Karplus has often cited Richard Feynman's (1918-88) prescient statement:

Certainly no subject or field is making more progress on so many fronts at the present moment, than biology, and if we were to name the most powerful assumption of all, which leads one on and on in an attempt to understand life, it is that all things are made of atoms, and that everything that liv-

\footnotetext{
47 Schatz (2000, p. 271).

48 Schatz (2000, p. 270).

49 Karplus by no means abandoned quantum chemistry after becoming interested in the $\left(\mathrm{H}, \mathrm{H}_{2}\right)$ exchange reaction. Instead, he put significant efforts into many-body perturbation theory and in correlated systems well into the 1970's.
}

ing things do can be understood in terms of the jigglings and wigglings of atoms ${ }^{50}$.

Karplus' first biochemical study focused on a very simple protein, the bovine pancreatic trypsin inhibitor (BPTI) taken in isolation, without anything around it. Yet, proteins function in solutions, and therefore the next steps would necessarily be an approach to intramolecular motions, and eventually the behavior of a protein inside water ${ }^{51}$.

We should also note here that hemoglobin played an important role in directing Karplus' attention toward biology; hemoglobin occupied a great deal of his own energy during the time after the KPS study. Although he had already worked on the visual chromophore, stimulated especially by Ruth Hubbard (1924-2016) but also by George Wald (1906-97) during his undergraduate training at Harvard, the effort to try to understand cooperativity in hemoglobin offered a more relevant biological problem. Karplus embarked on this problem around 1971, after hearing a lecture on the $\mathrm{X}$-ray structures of the oxygenated and deoxygenated state of the tetramer by Max Perutz (1914-2002) at the Massachusetts Institute of Technology, and following a discussion of the experimental result he had with Perutz after the lecture. However, with the first simulation of a protein, BPTI, Karplus realized that such simulations can give insight beyond those available from experiments and that the combination of empirical energy functions and classical MD held great potential for the better understanding of proteins. For this realization, Karplus deserves recognition.

In his Nobel lecture, he quoted J. Andrew McCammon's (born 1947) Oral History, making us grasp their excitement at that time ${ }^{52}$. Karplus always perceived the continuity between the classical MD simulations of proteins with his previous $\left(\mathrm{H}, \mathrm{H}_{2}\right)$ reaction study. As he put it in a discussion with us, "if hydrogen, for which one would expect the largest quantum corrections, could be treated by classical mechanics, it should work for systems like proteins, which are composed mainly of $\mathrm{C}, \mathrm{N}$, and O, as well as H." Karplus embarked in this research program while holding a fundamentally powerful position at Harvard, both in economic terms and in terms of the level of researchers working for him - a position which allowed him to take risks and make considerable changes to his research interests.

\footnotetext{
50 Feynman et al. (1963), Vol. I, Part. 3.3 Biology (https:// feynmanlectures.caltech.edu/I_03.html). Karplus (2006, pp. 15-16). See also Karplus (2020, p. 128).

${ }^{51}$ Karplus' first simulation of a dipeptide solvated by explicit water molecules was done in collaboration with Peter J. Rossky and Aneesur Rahman. See Rossky et al. (1979).

52 Karplus (2014). Andrew McCammon joined Karplus' group in the mid-1970s and contributed to the atomistic study of the dynamics of proteins. Karplus (2006, p. 36). See also David (1995) available at https://mccammon.ucsd. edu/people/pi/interview.php\#tc3.
} 


\section{Discussion}

We should acknowledge that Karplus' achievements were the results of teamwork and that Porter and Sharma played crucial roles, with Porter in particular making important contributions. In 1970, Porter also co-authored a textbook with Karplus, Atoms and Molecules $^{53}$. Sharma, on the other hand, was not involved in collaborative research with Karplus and Porter after $1966^{54}$

There are a few significant lessons that can be gleaned from an analysis of the primary sources. First, Karplus' ability to shift topics and continually change universities helped him skillfully modulate and further his professional career. In fact, during each of these changes, Karplus' research found new energy, encountering new stimuli from colleagues and students, gaining new perspectives and finding new opportunities to express his own creativity; those changes, in short, were fundamental to the advancement of his career.

Karplus' success was definitely well deserved. Each time he adopted a new direction, he demonstrated a deep grasp of the new scientific problems he focused on, together with a keen sense of the opportunity this new direction provided. For instance, the constant availability of tools and knowledge that could be repurposed to attack a specific problem also provided an important factor in his advancement. Karplus has often expressed the view that it is fine to be enthralled by an important and difficult problem, but passion is not enough. The realm of all possible scientific questions is extremely vast, and some problems, although fascinating and of the highest significance, might simply be too complex at a given time. Trying to work on a problem that is not mature enough, without self-reflection, may likely result in "wasting one's time." This is why Karplus always picked important problems, issues that had reached a certain level of maturity, for which one could make a meaningful contribution within a reasonable amount of time. Recognizing these problems definitely required a special talent and a profound insight. Again, as already underlined, Karplus' success exploited the computational approach. While ascending his professional path, he perpetually embarked on more ambitious projects. In other words, he continued enhancing his target with broader and broader projects, eventually reconnecting with his "first love" while keeping the vow he had made at Oxford, his promise to impact scholars across disciplines. In fact, at Harvard he had even wanted to study the functioning of the brain, but he was never able to make this jump, as it was not the right time for this problem.

Another aspect to ponder is the level of risk Karplus confronted during these career shifts. Even if he assumed a certain amount of risk while shaping his research projects, we do not believe that he really risked his own

\footnotetext{
53 Karplus and Porter (1971).

54 The last collaborative paper involving also Sharma is Karplus et al. (1966).
}

career, at least no more than any other scholar might do. Although Karplus embraced a quasiclassical scattering study in a quantum world - a fact that might on its face seem brave and risky - this decision was the result of careful choices stemming from teamwork, and from the consideration that this was essentially the only feasible path to follow in the mid-1960s. Karplus' most open-ended period was probably at Oxford, when he was deeply considering his own future. From his own biography, we know that, as a postdoc, he clearly pondered which area to target and how to be influential within that area. He also considered which contributions would most benefit other scholars, including those from other disciplines. Later, at Columbia, Karplus' place was safe, respected, visible, and powerful. At Harvard, his situation grew even safer, notwithstanding the research projects he decided to embark on. Being a Harvard professor meant power, an abundance of researchers, freedom to take risky paths, and opportunities to develop creatively. Harvard, that is, constituted a huge opportunity for Karplus, allowing for substantial intellectual freedom. Even if other scientists did not like what Karplus was doing - especially when he embarked on the study of biomolecules from a classical standpoint - he never really grew worried; he was in a position to do virtually anything he wanted, within reason. From this perspective, it is interesting to note that his Harvard colleagues most interested in molecular collisions, Dudley Herschbach (born 1932) and William Klemperer (1927-2017), did not immediately pay attention to the KPS work. In communication with us, Karplus remarked that this work had not been directly encouraged by his experimental colleagues. In contrast, the work on the J-coupling at Urbana-Champaign received great attention from his colleagues, especially from Gutowsky and Charles Slichter (1924-2018). Eventually however, Herschbach became interested in Karplus' calculation of reaction cross sections and particularly in the application to molecular beam experiments.

All through his career, Karplus maintained a fairly large number of collaborators in his laboratory. The resource of a large group certainly contributed to his ability to venture into new research areas and vigorously pursue new projects. Nevertheless, this favorable situation did not stem from an unusual amount of funding. Many of his students and postdocs were supported by their own fellowships, e.g., from the NSF or the National Institutes of Health (NIH), and the salary of visiting scientists was normally covered. Through most of his career, Karplus only received a few long-term grants, from NSF, NIH and the Department of Energy (DOE), which he used mainly to support students ${ }^{55}$.

It is worth discussing the kind of creativity that characterized Karplus' approach. Karplus was not exactly an inventor of new fundamental ideas; he was rather a remarkably creative and pragmatic scientist who could advance something that was already "in the air,"

\footnotetext{
55 For over sixty-five years, Karplus supported about 250
} students, postdocs, and visiting scientists. His very first 
exploiting tools and methods that had already been established before him. In the case of the $\left(\mathrm{H}, \mathrm{H}_{2}\right)$ reaction, he made use of previous studies and understood that further intellectual breakthroughs could occur only by exploiting computer technologies to obtain a sufficiently realistic and accurate Born-Oppenheimer potential surface, by using which he computed the scattering process and compared the results directly with experiments. Even before, especially when he had turned to NMR studies, he had relied on contributions that had already been developed, as we've seen in the case of Lemieux, thus leading them to produce significant results. This is an essential difference between Karplus and, say, Feynman, who was an inventor of completely new ideas and tools. With this observation, we do not mean to diminish the merits of Karplus' own work; rather, we wish to underline how science proceeds through the force of very different types of intelligences, those who invent new ideas and conceive completely novel theoretical perspectives, and those who combine existing tools in new and creative ways, focusing on problems that are already at the forefront. We believe that Karplus belongs in this second category. Yet, for an efficient scientific development, both approaches are useful and important ${ }^{56}$. His contributions - and perhaps we should better say the contributions of the KPS collaboration - to the study of elementary reactions were instrumental in promoting further research lines, not to speak of the subsequent development of biochemistry by $\mathrm{MD}^{57}$.

A final point is whether Karplus' approach may be seen as paradigmatically unorthodox. Indeed, his quasiclassical scattering studies might be considered so, occurring as they did during a time when quantum mechanics was deemed "the only serious way" to approach theoretical chemistry. And observing this "unorthodox" approach may in fact provide meaningful perspectives on twentieth-century scientific knowledge production. As previously anticipated, the partial return to a quasiclassical approach, articulated in terms of scattering studies, was made possible after the quantum treatment of the potential energy in the BornOppenheimer approximation. In practice, a quasiclassical approach was the only possible way to proceed during the mid-1960s. We would have to wait until 1975 before Aron Kuppermann (1926-2011) and Schatz were to set up a fully quantum-mechanical scattering treat-

\section{Footnote 55 continued}

student was Robert Harris (born in 1936) at UrbanaChampaign in 1955. Karplus generally had long-term grants, one from the NSF and one from NIH, which he used for supporting students. As he remarked in a conversation with us, for a while the NSF grant was replaced by a DOE grant. See also Karplus (2006, p. 27).

56 In this context, it is worth nothing that Feynman, interested in computing, was essentially looking to quantum computing (a topic to which he contributed a little) while he was much less interested in computer simulations.

57 Schatz (2000, p. 271). ment $^{58}$. It is reasonable to believe that KPS would have adopted a full quantum approach if it had been practically feasible; in fact, the quasiclassical scattering studies represent an interesting singularity during a time when quantum approaches were most popular and trusted.

\section{Conclusions}

During the years 1964-65, Karplus and his coworkers made seminal contributions to the study of the $\left(\mathrm{H}, \mathrm{H}_{2}\right)$ collision-reaction, both in terms of the calculation of the potential energy (Born-Oppenheimer) surface for the reaction $(\mathrm{PK})$ and in terms of the scattering study (KPS). While the former relied on a quantum approach, the latter was based purely on classical trajectories. Those years reveal aspects that may help historians frame Karplus' overall approach to research, something we tried to articulate throughout this paper. In addition, while the 1964 PK potential energy surface still needed to be further improved to reach chemical accuracy as pointed out by Schatz, it was quite realistic and sufficiently accurate to set the stage for the 1965 KPS quasiclassical reactive-collision calculations that made direct comparisons with experimental measurements possible. For this reason, we believe that both the 1964 PK and 1965 KPS articles represented landmarks in the process of theoretical chemistry becoming computational.

Karplus' own research was made possible because of the use of computers, and his merit derives from his unconditioned trust in computing technologies. This puts him on a different level with respect to most of his colleagues at the time. Karplus was able to grasp the totality of an interesting scientific problem, skillfully tease out opportunities, and make creative use of existing tools. He was able to promptly change directions, to find new routes to follow, and to select problems that held the potential for remarkable, pioneering results. This professional agility calls to mind an image that Karplus himself describes in his biography, when referring to his days as a doctoral student at Caltech:

My parents had given me their old car as a graduation present, and several times during my Caltech career I drove across the country to our home in Newton, Massachusetts, for part of the summer. Each time I took a different route, once through Canada with visits to the Banff and Jasper National Parks, and another time through the Deep South ${ }^{59}$.

This passage reveals an affinity for constantly changing routes, exactly as Karplus did in his scientific life.

\footnotetext{
58 Kuppermann and Schatz (1975). The interested reader may also consider some of the contributions that immediately followed. See, for example, Schatz et al. (1975a, b) and Schatz and Kuppermann (1976a, b).

59 Karplus (2006, pp. 16-17).
} 
Acknowledgements This work has been made possible thanks to the support of the Neubauer Collegium for Culture and Society at the University of Chicago, and to the help provided by the Stevanovich Institute on the Formation of Knowledge. The authors are especially grateful to Martin Karplus, friend and maestro, for his generous availability throughout this study, and for permission to quote from his personal recollections. We also thank Marci Karplus for her assistance, Jonathan Baker for the editorial support, as well as Juan Fuentes for his help in the initial stages of this research.

Open Access This article is licensed under a Creative Commons Attribution 4.0 International License, which permits use, sharing, adaptation, distribution and reproduction in any medium or format, as long as you give appropriate credit to the original author(s) and the source, provide a link to the Creative Commons licence, and indicate if changes were made. The images or other third party material in this article are included in the article's Creative Commons licence, unless indicated otherwise in a credit line to the material. If material is not included in the article's Creative Commons licence and your intended use is not permitted by statutory regulation or exceeds the permitted use, you will need to obtain permission directly from the copyright holder. To view a copy of this licence, visit http://creativecomm ons.org/licenses/by/4.0/.

\section{References}

Blais, Normand C., and Don L. Bunker. 1962. Monte Carlo calculations. II. The reactions of alkali atoms with methyl iodide. J. Chem. Phys. 37 (11): 2713-2720.

Boys, Samuel Francis, and Isaiah Shavitt. 1959. University of Wisconsin Naval Research Laboratory Tech. Rept. WI8-AF-13.

Bradshaw, W.H., H.E. Conrad, E.J. Corey, I.C. Gunsalus, and Daniel Lednicer. 1959. Microbiological degradation of (+)-camphor. J. Am. Chem. Soc. 81 (20): 5507.

Conroy, H. 1960. Nuclear magnetic resonance in organic structural elucidation. In Advances in organic chemistry: methods and results, eds. R.A. Raphael, E.C. Taylor, and H.Wijnberg, vol. 2, 265-294. New York: Interscience Publisher.

David, Allison. 1995. Transcript of an Oral History Interview with J. Andrew McCammon, National Museum of American History, Smithsonian Institution, April 13.https://mccammon.ucsd.edu/people/pi/ interview.php\#tc3

Eyring, Henry, and Michael Polanyi. 1931. Über einfache Gasreaktionen. Z. Phys. Chem. B 12: 279-311.

Feynman, Richard P., R.B. Leighton, and Matthew Sands. 1963. The Feynman lectures on physics. Boston: Addison-Wesley.

Heitler, Walter, and Fritz London. 1927. Wechselwirkung neutraler Atome und homöopolare Bindung nach der Quantenmechanik. Z. Phys. 44 (6-7): 455-472.

Hirschfelder, Joseph O. 1938. The energy of the triatomic hydrogen molecule and ion, V. J. Chem. Phys. 6 (12): 795-806.
Hirschfelder, Joseph O., H. Eyring, and Bryan Topley. 1936. Reactions involving hydrogen molecules and atoms. $J$. Chem. Phys. 4 (3): 170-177.

Hirschfelder, Joseph O., H. Eyring, and Nathan Rosen. 1936. I. Calculation of energy of $\mathrm{H}_{3}$ molecule. J. Chem. Phys. 4 (2): 121-130.

Karplus, Martin 2020. Spinach on the ceiling: the multifaceted life of a theoretical chemist. London: World Scientific Publishing Europe Ltd.

Karplus, Martin, and Richard N. Porter. 1971. Atoms and molecules an introduction for students of physical chemistry. New York: W. A. Benjamin.

Karplus, Martin, R.N. Porter, and Ramesh D. Sharma. 1964. Dynamics of reactive collisions: The $\mathrm{H}+\mathrm{H}_{2}$ exchange reaction. J. Chem. Phys. 40 (7): 2033-2034.

Karplus, Martin, R.N. Porter, and Ramesh D. Sharma. 1965. Exchange reactions with activation energy. I. Simple barrier potential for $\left(\mathrm{H}, \mathrm{H}_{2}\right)$. J. Chem. Phys. 43 (9): 3259-3287.

Karplus, Martin, R.N. Porter, and Ramesh D. Sharma. 1966. Energy dependence of cross sections for hot tritium reactions with hydrogen and deuterium molecules. J. Chem. Phys. 45 (10): 3871-3873.

Karplus, Martin. 1959. Contact electron-spin interactions of nuclear magnetic moments. J. Chem. Phys. 30 (1): $11-15$.

Karplus, Martin. 1959. Interpretation of the electron-spin resonance spectrum of the methyl radical. J. Chem. Phys. 30 (1): 15-18.

Karplus, Martin. 1960. Theory of proton coupling constants in unsaturated molecules. J. Am. Chem. Soc. 82 (16): 4431-4432.

Karplus, Martin. 1960. Weak interactions in molecular quantum mechanics. Rev. Mod. Phys. 32 (2): 455-460.

Karplus, Martin. 1963. Vicinal proton coupling in nuclear magnetic resonance. J. Am. Chem. Soc. 85 (18): 28702871.

Karplus, Martin. 2006. Spinach on the ceiling: a theoretical chemist's return to biology. Ann. Rev. Biophys. Biomol. Struct. 35 (1): 1-47.

Karplus, Martin. 2014. Development of multiscale models for complex chemical systems: from $\mathrm{H}+\mathrm{H}_{2}$ to biomolecules (Nobel Lecture). Angew. Chem. Int. Ed. Engl. 53 (38): 9992-10005.

Kimball, George E., and John G. Trulio. 1958. Quantum mechanics of the $\mathrm{H}_{3}$ complex. J. Chem. Phys. 28 (3): 493-497.

Kuppermann, Aron, and George C. Schatz. 1975. Quantum mechanical reactive scattering: an accurate threedimensional calculation. J. Chem. Phys. 62 (6): 25022504.

Lewis, Gilbert N. 1916. The atom and the molecule. J. Am. Chem. Soc. 38 (4): 762-785.

London, Fritz. 1929. Quantenmechanische Deutung des Vorgangs der Aktivierung. Z. Elektrochem. Angew. Phys. Chem. 35 (9): 552-555.

Macuglia, Daniele, B. Roux, and Giovanni Ciccotti. 2020. Sense Experiences and "necessary simulations": four centuries of scientific change from Galileo to fundamental computer simulations. KNOW 4 (1): 63-87.

Orgel, L.E. 1973. The origins of life: molecules and natural selection. London: Chapman and Hall. 
Porter, Ronald N., and Martin Karplus. 1964. Potential energy surface for $\mathrm{H}_{3}$. J. Chem. Phys. 40 (4): 11051115.

Ransil, Bernard J. 1957. Application of configuration interaction to the $\mathrm{H}_{3}$ complex. J. Chem. Phys. 26 (4): 971.

Rich, A. 2019. The excitement of discovery:selected papers of Alexander Rich, eds. S. Zhang. Hackensack, NJ: World Scientific Publishing.

Rossky, Peter J., M. Karplus, and Aneesur Rahman. 1979. A model for the simulation of an aqueous dipeptide solution. Biopolymers 18 (4): 825-854.

Sato, Shin. 1955. A new method of drawing the potential energy surface. Bull. Chem. Soc. Jpn. 28 (7): 450-453.

Sato, Shin. 1955. On a new method of drawing the potential energy surface. J. Chem. Phys. 23 (3): 592-593.

Sato, Shin. 1955. Potential energy surface of the system of three atoms. J. Chem. Phys. 23 (12): 2465-2466.

Schatz, George C. 2000. Perspective on "Exchange reactions with activation energy. I. Simple barrier potential for (H, $\left.\mathrm{H}_{2}\right)$." Theor. Chem. Acc. 103 (3): 270-272.

Schatz, George C., and Aron Kuppermann. 1976. Quantum mechanical reactive scattering for three-dimensional atom plus diatom systems. I. Theor. J. Chem. Phys. 65 (11): 4642-4667.

Schatz, George C., and Aron Kuppermann. 1976. Quantum mechanical reactive scattering for three-dimensional atom plus diatom systems. II. Accurate cross sections for $\mathrm{H}+\mathrm{H}_{2}$. J. Chem. Phys. 65 (11): 4668-4692.
Schatz, George C., J.M. Bowman, and Aron Kuppermann. 1975. Exact quantum, quasiclassical, and semiclassical reaction probabilities for the collinear $\mathrm{F}+\mathrm{H}_{2} \rightarrow \mathrm{FH}+\mathrm{H}$ reaction. J. Chem. Phys. 63 (2): 674-684.

Schatz, George C., J.M. Bowman, and Aron Kuppermann. 1975. Exact quantum, quasiclassical, and semiclassical reaction probabilities for the collinear $\mathrm{F}+\mathrm{D}_{2} \rightarrow \mathrm{FD}+$ D reaction. J. Chem. Phys. 63 (2): 685-696.

Schrödinger, Erwin. 1926. Quantisierung als Eigenwertproblem. Ann. Phys. 79 (4): 361-376.

Shavitt, Isaiah. 1959. A calculation of the rates of the orthopara conversions and isotope exchanges in hydrogen. $J$. Chem. Phys. 31 (5): 1359-1367.

Snow, Richard, and Henry Eyring. 1957. Activated complex energies. J. Phys. Chem. 61 (1): 1-6.

Wall, Frederick T., L.A. Hiller Jr., and Jacob Mazur. 1958. Statistical computation of reaction probabilities. J. Chem. Phys. 29 (2): 255-263.

Weston Jr., Ralph E. 1959. $\mathrm{H}_{3}$ activated complex and the rate of reaction of hydrogen atoms with hydrogen molecules. J. Chem. Phys. 31 (4): 892-898. 